\title{
Association between diabetes distress and all-cause mortality in Japanese individuals with type 2 diabetes: a prospective cohort study (Diabetes Distress and Care Registry in Tenri [DDCRT 18])
}

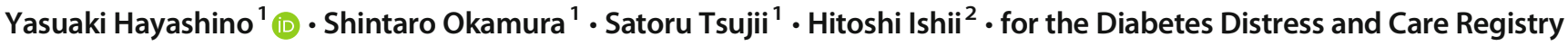 \\ at Tenri Study Group
}

Received: 15 March 2018 / Accepted: 14 May 2018 / Published online: 8 June 2018

(C) Springer-Verlag GmbH Germany, part of Springer Nature 2018

\begin{abstract}
Aims/hypothesis The absence of data on the direct association between diabetes-specific distress and all-cause mortality in individuals with diabetes prompted us to examine the temporal association between Problem Areas in Diabetes (PAID) survey scores and the subsequent risk of all-cause mortality in a cohort of individuals with type 2 diabetes.

Methods Longitudinal data from 3305 individuals with diabetes were obtained from a large Japanese diabetes registry. Independent correlations between quintiles of PAID total scores or PAID scores of $\geq 40$ and all-cause mortality (median follow-up of 6.1 years) were examined using Cox proportional hazards models with adjustment for potential confounders.

Results The study population included 1280 women and 2025 men with a mean age of 64.9 years, BMI of $24.6 \mathrm{~kg} / \mathrm{m}^{2}$ and $\mathrm{HbA}$ level of $58.7 \mathrm{mmol} / \mathrm{mol}(7.5 \%)$. In the multivariable-adjusted model, compared with the first quintile of PAID scores, the multivariable-adjusted HRs (95\% CIs) for all-cause mortality for the second to fifth quintiles were $1.11(0.77,1.60 ; p=0.56)$, $0.87(0.56,1.35 ; p=0.524), 0.95(0.63,1.46 ; p=0.802)$ and $1.60(1.09,2.36 ; p=0.016)$, respectively. Compared with a PAID score of $<40$, the multivariable-adjusted HR for all-cause mortality of those with a score of $\geq 10$ was $1.56(95 \%$ CI $1.17,2.08 ; p=0.002)$. In subgroup analyses, the association between PAID score and all-cause mortality was found in men (HR 1.76; 95\% CI 1.26, 2.46) but not in women (HR 1.09; 95\% CI 0.60, 2.00), with a significant interaction between diabetes distress and sex $(p=0.0336)$. Conclusions/interpretation We observed a significant positive association between high diabetes distress and all-cause mortality in men with diabetes.
\end{abstract}

Keywords Diabetes distress $\cdot$ Epidemiology $\cdot$ Human $\cdot$ Mortality $\cdot$ Problem Areas in Diabetes survey $\cdot$ Type 2 diabetes

\author{
Abbreviations \\ CRC Clinical research coordinator \\ IFCC International Federation of Clinical Chemistry \\ NGSP National Glycohemoglobin \\ Standardization Program \\ PAID Problem Areas in Diabetes
}

Members of the Diabetes Distress and Care Registry at Tenri Study Group are listed in the Appendix.

Yasuaki Hayashino

hayasino-y@umin.net

1 Department of Endocrinology, Tenri Hospital, 200 Mishima-cho, Tenri City, Nara 632-8552, Japan

2 Department of Diabetology, Nara Medical University, Kashihara, Nara, Japan

\section{Introduction}

Type 2 diabetes encompasses a complicated and heterogeneous group of metabolic alterations characterised by chronic hyperglycaemia. The prevalence of type 2 diabetes has dramatically increased over the past few decades worldwide [1], and is projected to increase further over the coming decades [2]. Type 2 diabetes and its complications contribute to a large portion of the burden of morbidity and disability worldwide. The 2016 Global Burden of Disease Study identified diabetes mellitus as the 12th major cause of reduced life expectancy, up from 23rd place in 1990 and 16th place in 2006 [3].

Self-management is the basis of diabetes care, and individuals with diabetes are expected to carry out daily selfmanagement activities to help avoid diabetes-related morbidity and mortality. Many people with diabetes feel burdened by the never-ending challenge of self-management and 


\section{Research in context}

\section{What is already known about this subject?}

- Many people with diabetes feel burdened by the never-ending challenge of self-management

- High levels of diabetes distress have been associated with poor glycaemic control and a high prevalence of complications in cross-sectional studies

\section{What is the key question?}

- Do high levels of diabetes distress lead to a higher risk of mortality in individuals with type 2 diabetes in the long term?

\section{What are the new findings?}

- High diabetes distress was associated with a higher risk of all-cause mortality in men with type 2 diabetes

How might this impact on clinical practice in the foreseeable future?

- We have provided new evidence on the importance of dealing with diabetes distress in clinical diabetes care

experience periods of frustration, anger, fear and helplessness, which are referred to collectively as diabetes distress [4]. The Diabetes Attitudes, Wishes and Needs 2 study reported that diabetes-related distress is common globally, and affects on average $44.6 \%$ of those with diabetes [5]. High levels of diabetes distress have been associated with poor glycaemic control and a high prevalence of complications in cross-sectional studies [6]; thus, we hypothesised that high levels of diabetes distress lead to a higher risk of mortality in the long term, and designed the present study to test this hypothesis.

We studied a cohort of Japanese individuals with type 2 diabetes from a large-scale Japanese diabetes registry to identify any associations between diabetes distress (measured using the Problem Areas in Diabetes [PAID] survey [7] at baseline) and the subsequent risk of all-cause mortality.

\section{Methods}

Participants Data were derived from a diabetes registry at Tenri Hospital, a regional tertiary-care teaching hospital in Japan. From October 2009 to August 2010, the registry recruited individuals diagnosed with diabetes who visited the outpatient clinic. In brief, this study is a cohort study aiming at evaluating the cross-sectional and prospective association between psychosocioeconomic factors, biomarkers, and the incidence of micro- and macrovascular complications in patients with diabetes [8]. Individuals diagnosed with impaired glucose tolerance and/or impaired fasting glucose using an oral glucose tolerance test were excluded. In total, 5133 individuals with diabetes visited the hospital, of whom 81 individuals were excluded from the study because of dementia $(n=15)$, mental retardation $(n=6)$, schizophrenia $(n=3)$, major depression $(n=2)$, visual disturbances $(n=35)$, poor general condition $(n=3)$ and other comorbidities $(n=17)$. Of the remaining 5052 individuals, 3898 (77.2\%) consented to participate in the study and were asked to complete the PAID survey at study registration, with the assistance of clinical research coordinators (CRCs). We further excluded individuals with diabetes other than type 2 diabetes $(n=199)$, those with a past history of any malignant neoplasms $(n=$ $365)$ and those who did not complete the PAID survey $(n=$ 29). In the end, data from 3305 individuals were used for the current analysis (1280 women and 2025 men). The ethics committee of Tenri Hospital approved this study. All participants gave their written informed consent before enrolment.

Data collection At baseline and every year thereafter, trained CRCs collected participant demographics from medical charts, including age, sex, body weight, duration of diabetes, past medical history (including microvascular and macrovascular complications) and treatment modalities. Prescription data were obtained electronically from the drug-ordering system. Laboratory tests (carried out during regular hospital visits) included random measurements (fasting was not required) of $\mathrm{HbA}_{1 \mathrm{c}}$ and the urinary albumin/creatinine ratio. On the same day as the laboratory tests, participants completed various selfadministered questionnaires (International physical activity questionnaire [9], questionnaire on hypoglycaemia frequency [10], and short form [SF]-8 [11]). $\mathrm{HbA}_{1 \mathrm{c}}$ levels were measured using the Japan Diabetes Society method [12], and then converted to the National Glycohemoglobin Standardization Program (NGSP) or the International Federation of Clinical Chemistry (IFCC) Working Group values [13]. Two trained CRCs independently input data from the medical charts or from written data to the data server at the Data Management Centre of Tenri hospital, and rechecked medical charts and original survey sheets for inconsistencies. 
Assessment of diabetes distress All participants completed a Japanese version of the PAID questionnaire embedded within a self-administered survey. Developed by Polonsky et al, the PAID questionnaire is a 20 item self-report that measures diabetes-related emotional distress with high reliability and clinical utility [7]. It was translated into Japanese by Ishii and the Japanese version shows good consistency and validity [14]. Questions such as 'From your own perspective, to what degree are the following diabetes-related issues currently a problem for you?' are rated using a five point scale for each item (ranging from $0=$ not a problem to $4=$ a serious problem). According to the authors' recommendation, we summed the item responses and multiplied by 1.25 to obtain a total score ranging from 0 to 100 .

Mortality Each year, researchers reviewed the list of registered participants and the vital status of each participant was checked using the information on medical charts. If a participant was no longer attending our hospital and the vital status was unknown then we conducted a follow-up using postal and telephone surveys. For each individual who had died, the underlying cause of death was classified on the basis of reviews of inpatient and outpatient medical records just preceding the death, the death certificate and other records (e.g. autopsy report), using the same methods as the Action to Control Cardiovascular Risk in Diabetes (ACCORD) study group [15]. The death certificate diagnosis was used when no other records were available. Two physicians reviewed all deaths and determined the underlying cause of death by consensus.

Statistical analysis Continuous variables are expressed as means and SDs or interquartile range, where indicated. Intergroup differences were evaluated using the unpaired Student's $t$ test for normally distributed variables, the MannWhitney $U$ test for variables with skewed distribution and the $\chi^{2}$ analysis for categorical variables.

We first looked for a linear association between the PAID total score and the risk of all-cause mortality. However, because the PAID score was not normally distributed in our sample, we categorised PAID total scores into quintiles and treated them as categorical variables. We used a Cox proportional hazards model to estimate HRs and their 95\% CIs for the all-cause mortality of each category compared with the first quintile. Each person-time was calculated from the date of the baseline survey to the date of death or of the last survey, whichever occurred first. We used two statistical models. The first model was adjusted for age and sex, while the second was multivariably adjusted for age, sex, BMI, $\mathrm{HbA}_{1 \mathrm{c}}$, type of diabetes therapy (diet only, oral medication only, insulin or glucagon-like receptor agonist injection), urinary albumin/ creatinine ratio $(<3,3-30$ or $\geq 30 \mathrm{mg} / \mathrm{mmol})$, history of any diabetic retinopathy, symptomatic diabetic neuropathy and past medical history (acute myocardial infarction, chronic stable angina, peripheral artery disease, leg ulceration, ischaemic stroke and haemorrhagic stroke).

Next, we performed an analysis using the known cut-off point for the PAID total score of $\geq 40$ to define diabetes distress, which has been found to be around 1 SD above the mean across different studies and has discriminative validity $[7,16]$. We used the above-mentioned two statistical models (age- and sex-adjusted or the multivariable-adjusted Cox proportional hazards model) to estimate HRs and their 95\% CIs for the all-cause mortality of participants with diabetes distress vs those without such distress. In addition, we performed subgroup analyses of the association between diabetes distress, defined by a PAID score of $\geq 40$, and diabetes by sex, age $(<65$ or $\geq 65$ years $), \mathrm{HbA}_{1 \mathrm{c}}$ ( $<58$ or $\left.\geq 58 \mathrm{mmol} / \mathrm{mol}[7.5 \%]\right)$ and diabetes therapy (diet only, oral medication only or any injection therapy). We selected the cut-off values of age and $\mathrm{HbA}_{1 \mathrm{c}}$ for the subgroup analysis because these values were close to the means. The statistical interactions were analysed using likelihood ratio tests, which compared the $-2 \log$ (likelihood) between two nested models: one considering the main effects alone and the other considering the main effects and additional interacting variables. We did not perform a cause-specific death analysis because of the very small number of outcomes in each death category. All reported $p$ values are two sided and $p<0.05$ was regarded as statistically significant, other than the test for interaction $(p<0.1)$. All analyses were performed using Stata/SE version 13.1 (Stata Corporation, College Station, TX, USA).

\section{Results}

Of the 3305 participants include in this analysis, 3012 completed laboratory tests and questionnaires in the second year, 2926 in the third year, 2742 in the fourth year, 2461 in the fifth year, 2359 in the sixth year and 2187 participants in the seventh year. Participants had a mean \pm SD age of $64.9 \pm 11.2$ years, BMI of $24.6 \pm 3.9 \mathrm{~kg} / \mathrm{m}^{2}$ and $\mathrm{HbA}_{1 \mathrm{c}}$ level of $58.7 \pm 12.7 \mathrm{mmol} / \mathrm{mol}$ $(7.5 \pm 1.2 \%)$. The median PAID total score was 29 , and the median scores for the first to fifth quintiles were 21, 25, 30, 39 and 55, respectively. Patient characteristics by PAID score quintile at baseline are shown in Table 1. We recorded 251 deaths during the median follow-up of 6.1 years (incidence ratio 14.0 per 1000 person-years) (Table 2). The most frequent cause of death was malignant neoplasia $(27.1 \%)$, followed by cardiovascular disease (19.9\%), infections (12.0\%) and ischaemic or haemorrhagic stroke $(4.8 \%)$. This order of ranking was similar to that found by a survey by the Japan Diabetes Society of the causes of death in 45,708 Japanese individuals with diabetes during the years 2001-2010 [17].

Compared with individuals in the first quintile of the PAID score, the age- and sex-adjusted HRs (95\% CI) for all-cause mortality for those in the second to fifth quintiles were 1.17 
Table 1 Participant characteristics based on PAID score quintiles at baseline

\begin{tabular}{|c|c|c|c|c|c|c|c|}
\hline Variable & All $(n=3305)$ & Q1 $(n=736)$ & Q2 $(n=720)$ & Q3 $(n=568)$ & Q4 $(n=662)$ & Q5 $(n=619)$ & $p$ value $^{\mathrm{a}}$ \\
\hline PAID total score & $29(23-41)$ & $21(20-22)$ & $25(24-26)$ & $30(29-32)$ & $39(36-42)$ & $55(50-62)$ & \\
\hline Age (years) & $64.9 \pm 11.2$ & $64.8 \pm 10.3$ & $63 \pm 11.3$ & $63 \pm 11.3$ & $62.7 \pm 12$ & $62.7 \pm 12$ & $<0.001$ \\
\hline Female & 38.7 & 36.3 & 36.3 & 41.0 & 37.9 & 49.6 & $<0.001$ \\
\hline BMI $\left(\mathrm{kg} / \mathrm{m}^{2}\right)$ & $24.6 \pm 3.9$ & $24.4 \pm 3.8$ & $24.8 \pm 4.1$ & $24.8 \pm 4.1$ & $25 \pm 4.3$ & $25 \pm 4.3$ & 0.0131 \\
\hline $\mathrm{HbA}_{1 \mathrm{c}}$ & & & & & & & $<0.001$ \\
\hline $\operatorname{IFCC}(\mathrm{mmol} / \mathrm{mol})$ & $58.7 \pm 12.7$ & $56.3 \pm 11.2$ & $57.0 \pm 11.7$ & $57.8 \pm 12.0$ & $59.9 \pm 51.1$ & $62.9 \pm 14.8$ & \\
\hline $\operatorname{NGSP}(\%)$ & $7.5 \pm 1.2$ & $7.4 \pm 1.1$ & $7.6 \pm 1.2$ & $7.6 \pm 1.2$ & $7.9 \pm 1.4$ & $7.9 \pm 1.4$ & \\
\hline Types of diabetes therapy & & & & & & & $<0.001$ \\
\hline Diet only & 61.8 & 66.9 & 66.9 & 62 & 58.5 & 49.1 & \\
\hline $\begin{array}{l}\text { Oral medication without } \\
\text { injection therapy }\end{array}$ & 14.8 & 11.5 & 11.5 & 14.6 & 18.9 & 20.7 & \\
\hline Insulin or GLP1-RA & 23.4 & 21.5 & 21.5 & 23.4 & 22.7 & 30.2 & \\
\hline Urinary albumin/creatinine ratio & & & & & & & 0.573 \\
\hline$<3 \mathrm{mg} / \mathrm{mmol}$ & 50.4 & 47.3 & 47.3 & 50.4 & 53.9 & 49.7 & \\
\hline $3-30 \mathrm{mg} / \mathrm{mmol}$ & 36.9 & 39.7 & 39.7 & 36.5 & 36.0 & 35.6 & \\
\hline$\geq 30 \mathrm{mg} / \mathrm{mmol}$ & 12.7 & 13.1 & 13.1 & 13.1 & 10.1 & 14.6 & \\
\hline Any diabetic retinopathy & 45.5 & 38.7 & 38.7 & 45.3 & 52.1 & 53.3 & 0.001 \\
\hline Symptomatic diabetic neuropathy & 9.3 & 9.0 & 9.0 & 8.6 & 9.1 & 10.2 & $<0.001$ \\
\hline \multicolumn{8}{|l|}{ Past medical history } \\
\hline Acute myocardial infarction & 9.3 & 9.0 & 9.0 & 8.6 & 9.1 & 10.2 & 0.905 \\
\hline Chronic stable angina & 11.7 & 11.9 & 11.9 & 12.7 & 12.4 & 10.7 & 0.71 \\
\hline Peripheral artery disease & 2.2 & 1.3 & 1.3 & 1.6 & 3.0 & 3.2 & 0.041 \\
\hline Leg ulceration & 1.0 & 1.0 & 1.0 & 1.2 & 0.8 & 1.1 & 0.897 \\
\hline Ischaemic stroke & 8.1 & 7.9 & 7.9 & 6.3 & 8.6 & 7.0 & 0.132 \\
\hline Haemorrhagic stroke & 1.2 & 1.4 & 1.4 & 0.9 & 0.8 & 0.8 & 0.314 \\
\hline
\end{tabular}

Data are median (IQR), means \pm SD or $\%$

GLP1-RA, glucagon-like peptide-1 receptor agonist; IQR, interquartile range

${ }^{\mathrm{a}} p$ value across groups

$(0.82,1.66 ; p=0.393), 0.84(0.54,1.31 ; p=0.445), 0.99$ $(0.66,1.49 ; p=0.954)$ and $1.71(1.18,2.47 ; p=0.004)$, respectively (Table 3 ), and we observed a significant linear trend $\left(p_{\text {trend }}=0.043\right)$. Adjusting for possible confounders slightly attenuated the association; the multivariable-adjusted HRs $(95 \% \mathrm{CI})$ for all-cause mortality were $1.11(0.77,1.60 ; p=$ $0.56), 0.87(0.56,1.35 ; p=0.524), 0.95(0.63,1.46 ; p=0.802)$ and $1.61(1.09,2.36 ; p=0.016)$, respectively, and the $p_{\text {trend }}$ went into the non-significant range $(p=0.089)$.
In the analysis using the cut-off point of PAID total score $\geq 40$ and comparing with the values for those without diabetes distress (i.e. PAID total score $<40$ ), the age- and sex-adjusted HR was 1.59 (95\% CI 1.21, 2.09; $p=0.001)$. After adjusting for possible confounders, we still observed a significant association between diabetes distress and all-cause mortality (HR 1.56; 95\% CI 1.17, 2.08; $p=0.002$ ).

The results of subgroup analyses are shown in Fig. 1. We observed a significant interaction $(p=0.0336)$ between
Table 2 PAID score quintiles and subsequent risk of all-cause mortality

\begin{tabular}{llllc}
\hline Quintile & Participants, $n$ & Person-years & Deaths, $n$ & Incidence ratio $^{\text {a }}(95 \%$ CI $)$ \\
\hline Q1 & 736 & 3926 & 69 & $17.6(13.9,22.3)$ \\
Q2 & 720 & 3816 & 59 & $15.5(12.0,20.0)$ \\
Q3 & 568 & 3127 & 30 & $9.6(6.7,13.7)$ \\
Q4 & 662 & 3703 & 38 & $10.3(7.5,14.1)$ \\
Q5 & 619 & 3293 & 55 & $16.7(12.8,21.8)$ \\
\hline
\end{tabular}

${ }^{\text {a }}$ Per 1000 person-years 
Table 3 Associations between PAID score (quintiles or score $<40$ or $\geq 40$ ) and subsequent risk of death

\begin{tabular}{|c|c|c|}
\hline HR (95\% CI) & Age- and sex-adjusted model & Multivariable-adjusted model ${ }^{\mathrm{a}}$ \\
\hline \multicolumn{3}{|c|}{ PAID total score (quintile) } \\
\hline $\mathrm{Q} 1(n=736)$ & Ref. & Ref. \\
\hline $\mathrm{Q} 2(n=720)$ & $1.17(0.82,1.66)$ & $1.11(0.77,1.60)$ \\
\hline Q3 $(n=568)$ & $0.84(0.54,1.31)$ & $0.87(0.56,1.35)$ \\
\hline $\mathrm{Q} 4(n=662)$ & $0.99(0.66,1.49)$ & $0.95(0.63,1.46)$ \\
\hline Q5 $(n=619)$ & $1.71(1.18,2.47)$ & $1.61(1.09,2.36)$ \\
\hline$p_{\text {trend }}$ & 0.043 & 0.089 \\
\hline \multicolumn{3}{|c|}{ PAID total score (two category) } \\
\hline$<40(n=2395)$ & Ref. & Ref. \\
\hline$\geq 40(n=910)$ & $1.59(1.21,2.09)$ & $1.56(1.17,2.08)$ \\
\hline$p$ value & 0.001 & 0.002 \\
\hline
\end{tabular}

${ }^{a}$ Adjusted for age, sex, BMI, $\mathrm{HbA}_{1 \mathrm{c}}$, types of diabetes therapy, urinary albumin/creatinine ratio, history of any diabetic retinopathy, symptomatic diabetic neuropathy and past medical history (acute myocardial infarction, chronic stable angina, peripheral artery disease, leg ulceration, ischaemic stroke and haemorrhagic stroke) diabetes distress and sex. Compared with those without diabetes distress, the HRs $(95 \% \mathrm{CI})$ for all-cause mortality for those with diabetes distress were $1.09(0.60,2.00)$ in women and $1.76(1.26,2.46)$ in men. We did not observe any interaction between diabetes distress and age $(p=0.1435), \mathrm{HbA}_{1 \mathrm{c}}$ levels $(p=0.35)$ or type of diabetes therapy $(p=0.1078)$.

\section{Discussion}

As far as we know, this is the first study to prospectively evaluate the association between diabetes distress and the subsequent risk of all-cause mortality in individuals with type 2 diabetes. The highest quintile of PAID total score (or those with diabetes distress defined as a PAID total score of $\geq 40$ ) was significantly associated with a higher risk of all-cause mortality than the lowest quintile of PAID score. Furthermore, we found this association only in men with type 2 diabetes, and not in women.

Clinically, dealing with diabetes distress is important because it can present a psychological obstacle to effective selfmanagement behaviours and therefore to achieving good glycaemic control [18, 19]. Several studies have evaluated the association between diabetes distress and glycaemic control or diabetes complications in individuals with diabetes. In one such study that looked at 3489 Japanese individuals with type 1 or type 2 diabetes, the PAID score quartile was significantly associated with poor glycaemic control $\left(\mathrm{HbA}_{1 \mathrm{c}}\right.$ $\geq 53 \mathrm{mmol} / \mathrm{mol}[7.0 \%]$ ), with a significant linear trend $(p=$ 0.03 ) even after adjusting for possible confounders [8]. A similar association has been observed in other regions [6, $20]$ and in studies using other measures of diabetes distress

\begin{tabular}{|c|c|c|c|}
\hline Subgroup & & $\mathrm{HR}(95 \% \mathrm{Cl})$ & $p$ value for interactio \\
\hline Sex & & & 0.0336 \\
\hline Female & - & $1.09(0.60,2.00)$ & \\
\hline Male & $\rightarrow$ & $1.76(1.26,2.46)$ & \\
\hline Age (years) & & & 0.1435 \\
\hline$<65$ & $\longrightarrow$ & $1.94(1.05,3.58)$ & \\
\hline$\geq 65$ & - & $1.20(0.86,1.66)$ & \\
\hline $\mathrm{HbA}_{1 \mathrm{c}}$ & & & 0.3500 \\
\hline$<58 \mathrm{mmol} / \mathrm{mol}(7.5 \%)$ & $\rightarrow$ & $1.75(1.20,2.57)$ & \\
\hline$\geq 58 \mathrm{mmol} / \mathrm{mol}(7.5 \%)$ & 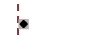 & $1.10(1.08,1.13)$ & \\
\hline Diabetes therapy & & & 0.1078 \\
\hline Diet only & $\longrightarrow$ & $1.69(0.90,2.55)$ & \\
\hline Oral glucose-lowering agent only & $\longrightarrow$ & $1.89(0.79,4.55)$ & \\
\hline Injection therapy & 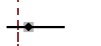 & $1.20(0.76,1.92)$ & \\
\hline
\end{tabular}

Fig. 1 Associations between the PAID total score ( $<40$ vs $\geq 40$ ) and allcause mortality in subgroups. A total of 3305 participants were included in the analysis. HRs and 95\% CIs were estimated using Cox regression models adjusted for age, sex, BMI, $\mathrm{HbA}_{1 \mathrm{c}}$, types of diabetes therapy, urinary albumin/creatinine ratio, history of any diabetic retinopathy, symptomatic diabetic neuropathy and past medical history (acute myocardial infarction, chronic stable angina, peripheral artery disease, leg ulceration, ischaemic stroke or haemorrhagic stroke). The $p$ values for interaction were obtained using likelihood ratio testing 
(Diabetes Distress Scale) [20]. However, these studies were limited by their cross-sectional design. One recently published study found that diabetes distress (defined by a PAID score of $\geq 40$ ) was not associated with worsening $\mathrm{HbA}_{1 \mathrm{c}}$ levels in individuals with type 2 diabetes [21]. This study was limited by a very short follow-up duration of only 2 years, and further evidence is needed to evaluate an association between diabetes distress and glycaemic control.

In a cross-sectional study of 2374 individuals with type 2 diabetes, a PAID-5 score of $\geq 40$ was associated with a higher prevalence of diabetes complications [6]. Because this was a cross-sectional study, however, it is possible that reverse causation applied and that individuals with diabetes complications were distressed. Two prospective studies have evaluated the association between distress and diabetes complications in individuals with diabetes. One found that general (not diabetes-specific) distress, as measured by the Mental Health Inventory 5, was associated with a 1.8-fold higher risk of mortality and a 1.7-fold higher risk of having a cardiovascular event in 1533 Swedish individuals with type 2 diabetes during a mean follow-up of 5.4 years [22]. The other prospective study evaluated the association between diabetes-specific distress, measured using PAID, and the subsequent risk of diabetic complications among individuals in the UK, and found that a PAID score of $\geq 40$ was not associated with microvascular and macrovascular complications in individuals with type 2 diabetes during the 2-year follow-up [21]. However, we believe that 2 years is too short a period to evaluate the effect of complications, and further studies are needed to examine the prospective association between diabetes distress and diabetes complications.

In our subgroup analysis, we observed a significant association of diabetes distress with all-cause mortality in men, but not in women. Few studies have evaluated sex differences in the association between psychological factors and mortality, but one meta-analysis that evaluated mortality associated with depression found the association to be higher in men [23], similar to our finding. The meta-analysis included 41,331 participants from 13 studies, and found that depression was associated with an increased risk of mortality in both men (RR 2.04 [95\% CI 1.76, 2.37]) and women (RR 1.55 [95\% CI $1.32,1.82])$ with a significant interaction $(p=0.015)$ [23]. Compared with depressed women, depressed men had a 1.97-fold higher risk of mortality (95\% CI 1.63, 2.37). The mechanism explaining this difference is unclear; it is possible that lifestyle or behavioural factors may be contributing.

We are aware of the limitations of our study. First, being an epidemiological study, residual confounders may exist for the association of diabetes distress with all-cause mortality. Because the number of deaths was relatively small, we were not able to perform cause-specific analysis for the association between diabetes distress and all-cause mortality; we hope a longer duration of follow-up of our cohort will make this possible. Another possible limitation is that we did not adjust for depression in the multivariable-adjusted model; however, diabetes distress and depression are different constructs, and thus we believe that adjusting for depression would not influence our results [4]. In addition, deriving data from the registry of a single diabetes centre raises concerns regarding the generalisability of our results, particularly for the multiethnic North American and European populations.

In conclusion, among individuals with type 2 diabetes who were experiencing high levels of diabetes distress, only men displayed a higher risk of all-cause mortality compared with non-distressed individuals. Further studies will be needed to uncover the mechanism of this association between diabetes distress and mortality among men with type 2 diabetes.

Acknowledgements We would especially like to thank Y. Moritsuji, Y. Fujita, N. Nakamura and Y. Sakamoto (Department of Endocrinology, Tenri Hospital) for their clerical support. The authors would like to thank Enago (www.enago.jp) for English language review.

Data availability The data that support the findings of this study are available on request from the corresponding author. The data are not publicly available, because they contain information that could compromise participant privacy.

Funding This study was partially supported by the Manpei Suzuki Diabetes Foundation and the Japan Society for the Promotion of Science (JSPS) KAKENHI (grant number 25460641). These organisations played no role in the study design or conduct, data collection, analysis or interpretation, or preparation, review or approval of the manuscript.

Duality of interest The authors declare that there is no duality of interest associated with this manuscript.

Contribution statement $\mathrm{YH}$ and $\mathrm{SO}$ searched the literature, conceived the study, analysed the data, interpreted the results, wrote the first draft of most sections of the report, obtained funding, collected the data, revised the report and participated in the writing of the report. YH was project coordinator. SO, ST and HI organised and supervised the study, interpreted the results and revised the report. All authors finally approved the version to be published. YH is the guarantor of this work and, as such, had full access to all the data in the study and takes responsibility for the integrity of the data and the accuracy of the data analysis.

\section{Appendix}

\section{Members of the Diabetes Distress and Care Registry in Tenri Study Group:}

Hitoshi Ishii, Hirohito Kuwata and Tsuyoshi Mashitani (Department of Diabetology, Nara Medical University, Kashihara, Nara, Japan); Satoru Tsujii, Shintaro Okamura, Yasuaki Hayashino, Miyuki Furuya, Kiyoko Takano, Kentaro Kurosawa, Yui Sakuramachi and Masako Kitatani (Department of Endocrinology, Tenri Hospital, Tenri, Nara, Japan); Satoshi Matsunaga (Department of Hematology, 
Endocrinology and Metabolism, Niigata University Faculty of Medicine, Niigata, Japan); Yaeko Kondo and Naotaka Fujita (Department of Diabetes and Clinical Nutrition, Kyoto University, Kyoto, Japan); Rei Ueda (Second Department of Internal Medicine, Faculty of Medicine, University of the Ryukyus, Naha, Okinawa, Japan); Rie Kurokawa (Sawa Hospital, Osaka, Japan); and Masami Tanaka (Division of Endocrinology, Metabolism and Nephrology, Department of Internal Medicine, Keio University School of Medicine, Tokyo, Japan).

\section{References}

1. Kahn SE, Cooper ME, Del Prato S (2014) Pathophysiology and treatment of type 2 diabetes: perspectives on the past, present, and future. Lancet 383:1068-1083

2. Whiting DR, Guariguata L, Weil C, Shaw J (2011) IDF diabetes atlas: global estimates of the prevalence of diabetes for 2011 and 2030. Diabetes Res Clin Pract 94:311-321

3. GBD 2016 DALYs and HALE Collaborators (2017) Global, regional, and national disability-adjusted life-years (DALYs) for 333 diseases and injuries and healthy life expectancy (HALE) for 195 countries and territories, 1990-2016: a systematic analysis for the Global Burden of Disease Study 2016. Lancet 390:1260-1344

4. Gonzalez JS, Fisher L, Polonsky WH (2011) Depression in diabetes: have we been missing something important? Diabetes Care 34 : 236-239

5. Nicolucci A, Kovacs Burns K, Holt RI et al (2013) Diabetes Attitudes, Wishes and Needs second study (DAWN2 ${ }^{\mathrm{TM}}$ ): crossnational benchmarking of diabetes-related psychosocial outcomes for people with diabetes. Diabet Med 30:767-777

6. Pintaudi B, Lucisano G, Gentile S et al (2015) Correlates of diabetes-related distress in type 2 diabetes: findings from the benchmarking network for clinical and humanistic outcomes in diabetes (BENCH-D) study. J Psychosom Res 79:348-354

7. Polonsky WH, Anderson BJ, Lohrer PA et al (1995) Assessment of diabetes-related distress. Diabetes Care 18:754-760

8. Hayashino Y, Okamura S, Matsunaga S, Tsujii S, Ishii H, Tenri Cohort Study Group (2012) The association between problem areas in diabetes scale scores and glycemic control is modified by types of diabetes therapy: diabetes distress and care registry in Tenri (DDCRT 2). Diabetes Res Clin Pract 97:405-410

9. Craig CL, Marshall AL, Sjöström M et al (2003) International physical activity questionnaire: 12-country reliability and validity. Med Sci Sports Exerc 35:1381-1395

10. Hayashino Y, Tsujii S, Ishii H, Diabetes Distress and Care Registry at Tenri Study Group (2013) High frequency of non-nocturnal hypoglycemia was associated with poor sleep quality measure by Pittsburg Sleep Quality Index in patients with diabetes receiving insulin therapy: Diabetes Distress and Care Registry at Tenri (DDCRT 4). Exp Clin Endocrinol Diabetes 121:628-634

11. Ware JE, Kosinski M, Dewey JE, Gandek B (2001) How to score and interpret single-item health status measures: a manual for users of the SF-8 health survey. QualityMetric, Lincoln

12. Committee of the Japan Diabetes Society on the Diagnostic Criteria of Diabetes Mellitus, Seino Y, Nanjo K et al (2010) Report of the committee on the classification and diagnostic criteria of diabetes mellitus. J Diabetes Investig 1:212-228

13. International Federation of Clinical Chemistry (IFCC) Working Group (2010) Standardization of HbA1c. Available at http://www. ngsp.org/docs/ifccstd.pdf. Accessed 15 Mar 2018

14. Ishii H (2000) Psycho-behavioral problems in diabetes treatment. J Japan Diab Soc 43:13-16 [article in Japanese]

15. The ACCORD Study Group, Buse JB, Bigger JT et al (2007) Action to Control Cardiovascular Risk in Diabetes (ACCORD) trial: design and methods. Am J Cardiol 99:21i-33i

16. Welch GW, Jacobson AM, Polonsky WH (1997) The Problem Areas in Diabetes Scale. An evaluation of its clinical utility. Diabetes Care 20:760-766

17. Nakamura J, Kamiya H, Haneda M et al (2017) Causes of death in Japanese patients with diabetes based on the results of a survey of 45,708 cases during 2001-2010: Report of the Committee on Causes of Death in Diabetes Mellitus. J Diabetes Investig 8:397410

18. Schmitt A, Reimer A, Kulzer B, Haak T, Gahr A, Hermanns N (2014) Assessment of diabetes acceptance can help identify patients with ineffective diabetes self-care and poor diabetes control. Diabet Med 31:1446-1451

19. Schmitt A, Reimer A, Kulzer B, Haak T, Gahr A, Hermanns N (2015) Negative association between depression and diabetes control only when accompanied by diabetes-specific distress. J Behav Med 38:556-564

20. Fisher L, Glasgow RE, Strycker LA (2010) The relationship between diabetes distress and clinical depression with glycemic control among patients with type 2 diabetes. Diabetes Care 33:1034 1036

21. Ismail K, Moulton CD, Winkley K et al (2017) The association of depressive symptoms and diabetes distress with glycaemic control and diabetes complications over 2 years in newly diagnosed type 2 diabetes: a prospective cohort study. Diabetologia 60:2092-2102

22. Dalsgaard EM, Vestergaard M, Skriver MV et al (2014) Psychological distress, cardiovascular complications and mortality among people with screen-detected type 2 diabetes: follow-up of the ADDITION-Denmark trial. Diabetologia 57:710-717

23. Cuijpers P, Vogelzangs N, Twisk J, Kleiboer A, Li J, Penninx BW (2014) Is excess mortality higher in depressed men than in depressed women? A meta-analytic comparison. J Affect Disord 161:47-54 